\title{
RESEARCH
}

Open Access

\section{NLRP7 deubiquitination by USP10 promotes tumor progression and tumor- associated macrophage polarization in colorectal cancer}

Bing $\mathrm{Li}^{1,2+}$, Zhi-Peng Qi ${ }^{1,2+}$, Dong-Li He ${ }^{3,4+}$, Zhang-Han Chen ${ }^{1,2+}$, Jing-Yi Liu' ${ }^{1,2}$, Meng-Wai Wong ${ }^{3}$, Jia-Wei Zhang ${ }^{4}$, En-Pan Xu ${ }^{1,2}$, Qiang Shi ${ }^{1,2}$, Shi-Lun Cai ${ }^{1,2}$, Di Sun ${ }^{1,2}$, Li-Qing Yao ${ }^{1,2}$, Ping-Hong Zhou ${ }^{1,2^{*}}$ and Yun-Shi Zhong ${ }^{1,2^{*}}$ (D)

\begin{abstract}
Background: NOD-like receptors affect multiple stages of cancer progression in many malignancies. NACHT, LRR, and PYD domain-containing protein 7 (NLRP7) is a member of the NOD-like receptor family, although its role in tumorigenesis remains unclear. By analyzing clinical samples, we found that NLRP7 protein levels were upregulated in colorectal cancer (CRC). We proposed the hypothesis that a high level of NLRP7 in CRC may promote tumor progression. Here, we further investigated the role of NLRP7 in CRC and the underlying mechanism.

Methods: NLRP7 expression in human CRC and adjacent non-tumorous tissues was examined by quantitative real-time polymerase chain reaction (qRT-PCR), western blotting, and immunohistochemistry. The effect of NLRP7 in CRC progression was investigated in vitro and in vivo. Proteins interacting with NLRP7 were identified by immunoprecipitation and mass spectrometry analysis while immunofluorescence staining revealed the cellular location of the proteins. Cellular ubiquitination and protein stability assays were applied to demonstrate the ubiquitination effect on NLRP7. Cloning and mutagenesis were used to identify a lysine acceptor site that mediates NLRP7 ubiquitination. Cytokines/chemokines affected by NLRP7 were identified by RNA sequencing, QRT-PCR, and enzyme-linked immunosorbent assay. Macrophage phenotypes were determined using qRT-PCR, flow cytometry, and immunohistochemistry.

(Continued on next page)
\end{abstract}

\footnotetext{
* Correspondence: zhou.pinghong@zs-hospital.sh.cn;

zhongyunshi@yahoo.com

${ }^{\dagger}$ Bing Li, Zhi-Peng Qi, Dong-Li He and Zhang-Han Chen contributed equally to this work and should be considered co-first authors.

${ }^{1}$ Endoscopy Center, Zhongshan Hospital of Fudan University, 180 Fenglin Road, Shanghai 20032, People's Republic of China

Full list of author information is available at the end of the article
}

C C The Author(s). 2021 Open Access This article is licensed under a Creative Commons Attribution 4.0 International License, which permits use, sharing, adaptation, distribution and reproduction in any medium or format, as long as you give appropriate credit to the original author(s) and the source, provide a link to the Creative Commons licence, and indicate if changes were made. The images or other third party material in this article are included in the article's Creative Commons licence, unless indicated otherwise in a credit line to the material. If material is not included in the article's Creative Commons licence and your intended use is not permitted by statutory regulation or exceeds the permitted use, you will need to obtain permission directly from the copyright holder. To view a copy of this licence, visit http://creativecommons.org/licenses/by/4.0/. The Creative Commons Public Domain Dedication waiver (http://creativecommons.org/publicdomain/zero/1.0/) applies to the data made available in this article, unless otherwise stated in a credit line to the data. 
(Continued from previous page)

Results: NLRP7 protein levels, but not mRNA levels, were upregulated in CRC, and increased NLRP7 protein expression was associated with poor survival. NLRP7 promoted tumor cell proliferation and metastasis in vivo and in vitro and interacted with ubiquitin-specific protease 10, which catalyzed its deubiquitination in CRC cells. NLRP7 stability and protein levels in CRC cells were modulated by ubiquitination and deubiquitination, and NLRP7 was involved in the ubiquitin-specific protease 10 promotion of tumor progression and metastasis in CRC. K379 was an important lysine acceptor site that mediates NLRP7 ubiquitination in CRC cells. In CRC, NLRP7 promoted the polarization of pro-tumor M2like macrophages by inducing the secretion of $C-C$ motif chemokine ligand 2. Furthermore, NLRP7 promoted NF-KB nuclear translocation and activation of C-C motif chemokine ligand 2 transcription.

Conclusions: We showed that NLRP7 promotes CRC progression and revealed an as-yet-unidentified mechanism by which NLRP7 induces the polarization of pro-tumor M2-like macrophages. These results suggest that NLRP7 could serve as a biomarker and novel therapeutic target for the treatment of CRC.

Keywords: Colorectal cancer, NOD-like receptors, NLRP7, Deubiquitination, Tumor-associated macrophages

\section{Background}

Colorectal cancer (CRC) is one of the most lethal carcinomas, and its incidence is increasing worldwide [1]. Despite an increase in the five-year net survival rates over the past few decades, the mortality rate of CRC remains high, which is mainly due to recurrence and distant organ metastasis $[2,3]$. Therefore, exploring the molecular mechanisms that drive tumor initiation and progression in CRC is critical for developing novel preventive and therapeutic strategies against this disease.

NOD-like receptors (NLRs), which were originally studied as critical regulators of the immune response, serve as pattern recognition receptors that specifically recognize pathogen-associated molecular patterns (PAMPs) [4]. Recent evidence supports the concept that innate immune signaling plays an important role in cancer progression [5, 6]. A significant role for members of the NLR family has been revealed as contributing either directly or indirectly to a variety of traits associated with cancer, including inflammation, cell death, tumor growth, angiogenesis, invasion, and metastasis [7-10]. In terms of functionality, some members can be initiators of the inflammasome pathway, which canonically activates caspase- 1 , and IL- $1 \beta$ and IL- 18 thereafter $[11,12]$. However, some other NLRs regulate diverse biological pathways associated with both inflammation and tumorigenesis through a non-inflammasome pathway [13-15]. For example, the NLRP3 inflammasome suppresses liver metastasis in CRC by IL-18 signaling [16]. However, NLRP3 upregulation in CRC cells can also promote epithelial-mesenchymal transition in an inflammasome-independent manner [15]. Therefore, in view of the multiple effects of NLR family members in cancer cells, a comprehensive understanding is needed of the multiple signaling pathways in which they are involved.

Among all members of the NLR family, NLRP7 and its function are poorly understood. Previous studies of NLRP7 have investigated effects on trophoblast lineage differentiation and contributions to hydatidiform mole development in abnormal human pregnancies [17, 18]. In a new area of research, a correlation between the NLRP7 gene and ulcerative colitis (UC) was recently reported, and NLRP7 expression was found to be upregulated in the intestinal mucosa of patients with inflammatory bowel disease (IBD) $[19,20]$. Considering that IBD patients have an increased risk of CRC and the function of NLRP7 in cancers remains unclear [21], we decided to explore the contribution of NLRP7 to colorectal tumorigenesis.

In this study, we found that NLRP7 protein levels, but not mRNA levels, were upregulated in CRC by analyzing clinical samples. We then proposed the hypothesis that a high level of NLRP7 in CRC may promote tumor progression, requiring further investigation. The function of NLRP7 in promoting tumor cell proliferation and metastasis was confirmed. Furthermore, we found that NLRP7 in CRC cells interacted with ubiquitin-specific protease 10 (USP10), which mediated its deubiquitination, thereby increasing its protein stability. In addition, NLRP7 promoted the polarization of pro-tumor M2-like macrophages in CRC by inducing the secretion of C-C motif chemokine ligand 2 (CCL2) through the activation of the NF- $\mathrm{kB}$ signaling pathway. Thus, we demonstrated that NLRP7 can promote both tumor progression and tumor-associated macrophage polarization in colorectal cancer.

\section{Methods}

\section{Cell culture and reagents}

HCT116 and HT29 cells were cultured in McCoy's 5A medium (GibCo) supplemented with 10\% FBS. DLD-1 and THP-1 cells were grown in RPMI 1640 (GibCo) with 10\% FBS. LoVo, SW480, and SW620 cells were cultured in DMEM (GibCo) high-glucose medium with $10 \%$ FBS. All cell lines were purchased from Chinese Academy of Sciences Cell Bank (Shanghai, China). THP- 
1 cells were differentiated into macrophages by exposure to $200 \mathrm{ng} / \mathrm{ml}$ phorbol-12-myristate-13-acetate (PMA) (Sigma-Aldrich) for $48 \mathrm{~h} \mathrm{[22].}$

\section{Human tissue specimens and immunohistochemical analysis}

Thirty pairs of fresh primary CRC and corresponding non-tumorous tissues were collected immediately after surgical resection at Zhongshan Hospital, Fudan University (Shanghai, China). Another 115 formalinfixed and paraffin-embedded (FFPE) CRC samples and adjacent non-tumorous tissue samples were also obtained from Zhongshan Hospital. Written informed consent was obtained from each patient, and the investigation was approved by the Ethics Committee of Zhongshan Hospital. Tumor-node-metastasis (TNM) staging was performed according to American Joint Committee on Cancer (AJCC) standards [23].

Consecutive sections of FFPE tumors were subjected to immunohistochemistry (IHC) staining using rabbit polyclonal anti-NLRP7 (Abcam, 1:50) and anti-USP10 (Abcam, 1:50) antibodies. A DAB substrate kit was used according to the manufacturer's instructions. The results were scored by two pathologists with no prior knowledge of patient characteristics. Corresponding to the percentage of immune-reactive tumor cells $(0,1-5,6-$ $25,26-75$, and $76-100 \%$, respectively), the staining extent score was on a scale of $0-4$. The staining intensity was scored as negative $($ score $=0)$, weak $($ score $=1)$, medium (score $=2$ ), or strong ( the staining extent score by the intensity score, a score ranging from 0 to 12 was calculated.

\section{Lentiviral-mediated NLRP7 overexpression and knockdown}

A lentiviral construct carrying NLRP7 (GeneChem, China) was packaged in 293FT cells. Virus-containing supernatants were collected and stably transfected into SW480 and HT29 cells selected by puromycin (Sigma-Aldrich). Empty vector transfected cells were used as controls. Two lentiviral constructs containing short hairpin RNAs (shRNA) were purchased from GeneChem to specifically establish NLRP7 knockdown cell lines. The shNLRP7 construct or the negative control plasmid was transfected into the 293FT cell line, and virus-containing supernatants were collected for subsequent transduction into HCT116 cells and DLD-1 cells. Puromycin (Sigma-Aldrich) was used to select for stably transduced cells. The sequences of the two shRNAs against NLRP7 are as follows: 5'TTGCTGAAGAGGAAGATGTTA-3' and ${ }^{\prime}{ }^{\prime}$ TGGCAAGAAACTGGCAGAAAT-3'.
RNA isolation and quantitative real-time PCR (qRT-PCR) Total RNA was extracted using the TRIzol reagent (Invitrogen), and complementary DNA (cDNA) was synthesized using a reverse transcription-PCR kit (Roche) according to the manufacturer's instructions. qRT-PCR was performed with Maxima SYBR Green qPCR Master Mix (Applied Biosystems) on a QuantStudio 6 Flex Real-Time PCR System (Applied Biosystems). GAPDH was used as an internal control. The primers were purchased from Asia-Vector Biotechnology Company (Shanghai, China), and their sequences are listed in Additional file 1: Table S1.

\section{RNA sequencing}

After total RNA extraction from DLD-1 and SW480 cells, the RNA quality and quantity were assessed by Nano Drop and Agilent 2100 bioanalyzer (Thermo Fisher Scientific). Strand-specific library construction and sequencing of $\sim 100 \mathrm{M}$ paired end.

100-bp-long reads were performed at the BGI (Huada Genomics Institute Co. Ltd., Guangzhou, China). Differential expression analysis was performed using DESeq2 software [24]. GO and KEGG enrichment analysis of annotated differently expressed genes was performed by phyper based on hypergeometric test.

\section{Western blot (WB) analysis}

Quantified protein lysates were separated by sodium dodecyl sulfate-polyacrylamide gel electrophoresis (SDSPAGE) and transferred onto polyvinylidene difluoride membranes. Subsequently, the proteins were blocked with $5 \%$ bovine serum albumin for $1 \mathrm{~h}$ at room temperature and incubated in primary antibodies at $4{ }^{\circ} \mathrm{C}$ overnight. After washing with TBS-T, the membranes were incubated in horseradish peroxidase-conjugated secondary antibody for $2 \mathrm{~h}$. GAPDH and $\beta$-actin were used as loading controls. The antibodies used are listed in Additional file 1: Table S2, and unprocessed original scans of blots are provided in Additional file 2.

\section{Immunoprecipitation (IP) assay}

As described elsewhere [25], whole-cell extracts were

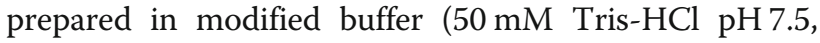
$150 \mathrm{mM} \mathrm{NaCl}, 5 \mathrm{mM} \mathrm{EDTA}$, and protease inhibitor cocktail) and incubated with the indicated antibodies or IgG at $4{ }^{\circ} \mathrm{C}$ for $2 \mathrm{~h}$, and then mixed with $100 \mu \mathrm{l}$ protein A/G agarose (Santa Cruz) overnight at $4{ }^{\circ} \mathrm{C}$. Beads were washed with PBS three times, and bound protein was denatured with $1 \times$ SDS sample buffer. Then, proteins were collected and analyzed by SDS-PAGE and western blotting. 


\section{Mass spectrometry analysis}

Similar to the IP assay, cellular protein extracts from HT29 cells were incubated with anti-Flag-NLRP7 followed by and protein A/G agarose beads. Recovered proteins associated with Flag-NLRP7 or IgG were resolved by gel electrophoresis. The bands specifically bind to Flag-NLRP7 were excised, and proteomics screening was accomplished by mass spectrometry analysis on a MALDI-TOF-MS instrument (Bruker Daltonics).

\section{Immunofluorescence (IF) staining}

Immunofluorescent staining was performed as described previously [26]. Briefly, cells were grown on glassbottom culture dishes until the experimental endpoint. Then, the cells were fixed with $4 \%$ paraformaldehyde, then washed three times with PBS, and probed with primary antibody against NLRP7, USP10, or NF- $\mathrm{kB}$ p65 at $4{ }^{\circ} \mathrm{C}$ overnight. After washing with PBS, the cells were incubated with fluorescence-conjugated secondary antibody (Invitrogen) for $30 \mathrm{~min}$. Next, the cells were washed twice with blocking solution, and once with PBS. Anti-fade DAPI solution (Life Technology) was then added for DNA staining. The cells were imaged using a confocal laser-scanning microscope. Images were analyzed for co-localization using CoLocalizer Pro Version 3.0.2 according to published protocols.

\section{Cloning and mutagenesis}

Full-length human NLRP7 cDNA and truncated NLRP7 were amplified from a human mRNA pool generated by RT-PCR. Site-directed mutagenesis (KKK275RRR, KRK288RRR, K374R, K379R, K461R, K484R, K502R, and $\mathrm{K} 532 \mathrm{R}$ ) was performed using the Site-Directed $\mathrm{Mu}$ tagenesis kit (Agilent) according to the manufacturer's protocol. The sequences of all primers are available in Additional file 1: Table S3. After cloning, cDNA was ligated into the hU6-MCS-CBh-gcGFP-IRES-puromycin vector purchased from GeneChem. The sequences were confirmed by direct sequencing.

\section{Flow cytometry}

Cells were collected and incubated at $4{ }^{\circ} \mathrm{C}$ with florescence-conjugated antibodies, and fixed and permeabilized with a fixation/permeabilization solution kit (BD Cytofix/Cytoperm) and 1\% paraformaldehyde (PFA). Flow cytometry results were analyzed with FlowJo software. Antibodies used are listed in Additional file 1: Table S2.

\section{Protein stability assay}

In different cell treatment groups, cycloheximide was added into culture medium and cell lysis was collected at $0,2,4,8,12,16$, or $24 \mathrm{~h}$ after the treatment of cycloheximide. Then, the protein was visualized using immunoblotting assays.

\section{Cellular ubiquitination assay}

As described previously, the cellular ubiquitination assays were conducted [27]. Briefly, IP assay was used and the immunoprecipitates were obtained by incubating cell lysates with anti-FLAG-NLRP7 and A/G agarose beads. The ubiquitinated FLAG-NLRP7 was visualized using immunoblotting assays with anti-HA.

\section{Enzyme linked immunosorbent assay (ELISA)}

The supernatant from CRC cells and serum from an animal model of metastasis was collected to detect the secretion level of CCL2 using an ELISA kit (R\&D Systems). ELISA was performed according to the manufacturer's instructions, and all experiments were performed in triplicate.

\section{Animal studies}

The animal experiment was performed with protocols approved by the Institutional Animal Care and Use Committee of Zhongshan Hospital of Fudan University. For in vivo tumorigenic experiments, HCT116 cells $(1 \times$ $10^{6}$ ) expressing sh-Ctrl, sh-NLRP7, sh-USP10, or shUSP10/NLRP7 were injected into the right dorsal flank of five-week-old Balb/c nude mice. Tumor formation in nude mice was monitored over a six-week period. The tumor volume was calculated with the following formula: tumor volume $=0.5 \times$ length $\times$ width $^{2}$. For in vivo metastasis assays, a small left abdominal flank incision was made, the spleen was exteriorized, and the prepared cells $\left(2 \times 10^{6}\right.$ cells $)$ were injected into the spleen with a $30-$ Gauge needle. Then, the injected spleen was returned to the abdomen and the wound was sutured with $6-0$ black silk. Six weeks later, all mice were sacrificed and necropsied for observation of visible metastatic lesions in the liver. In the metastasis animal assay, the peripheral blood of mice was collected at necropsy and stored at $-80^{\circ} \mathrm{C}$ until analysis. All primary and metastatic tumor tissues were harvested for further analysis by IHC staining.

\section{Statistical analysis}

Differences between groups were calculated using Student's t-test, the chi-square test, or the Fisher exact test. The probability of differences in overall survival (OS) was ascertained by the Kaplan-Meier method with a log-rank test for significance. Analyses were performed with SPSS version 22.0 and Graphpad Prism 7.0 statistical analysis software. Representative data are shown as the mean \pm SD. $P<0.05$ was considered statistically significant. 


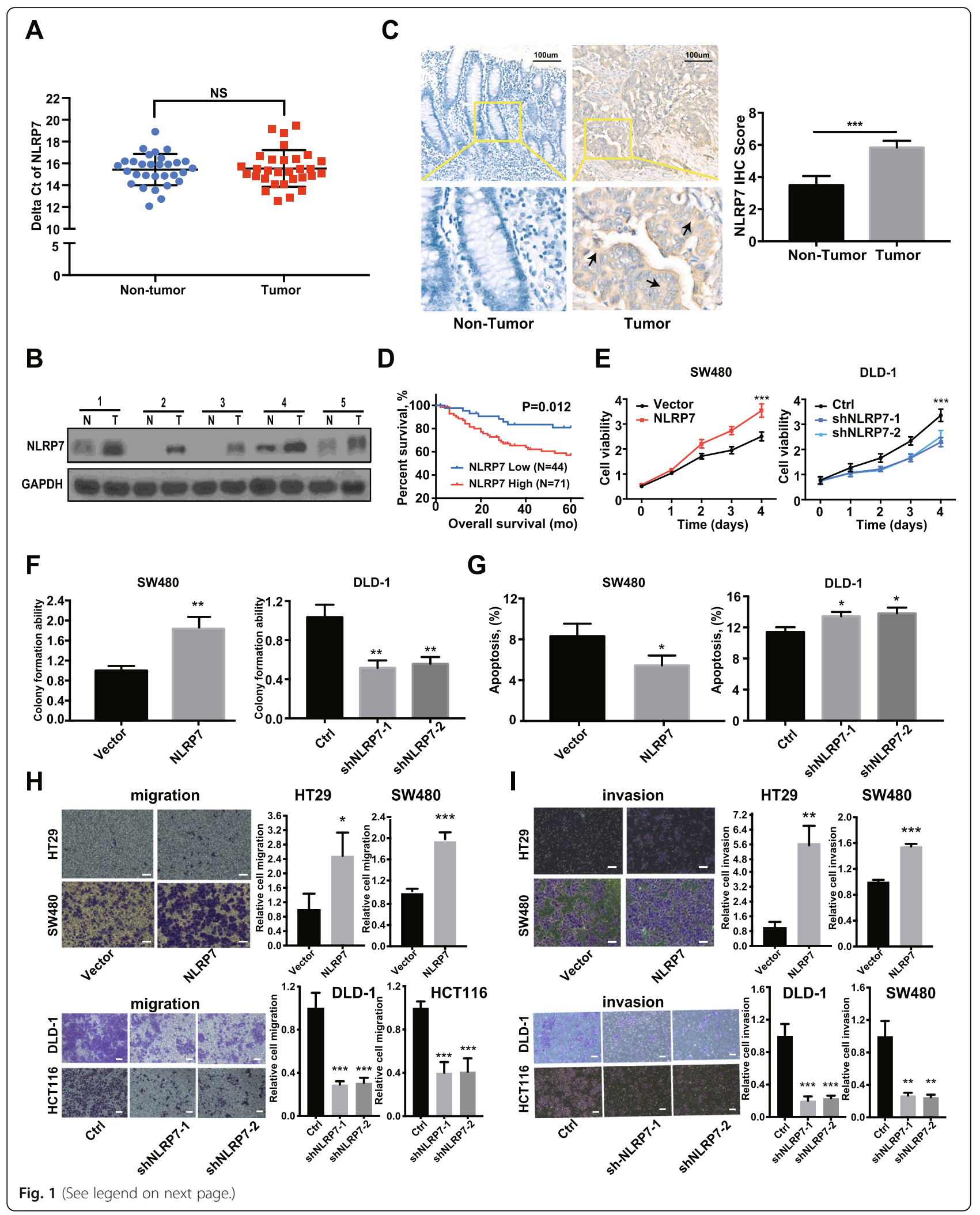


(See figure on previous page.)

Fig. 1 NLRP7 protein with high expression level in CRC promotes tumor cell proliferation and metastasis. a NLRP7 mRNA levels in CRC tissues and the normal colorectal mucosa measured by qRT-PCR. $\mathbf{b}$ The protein levels of NLRP7 in CRC tissues and paired normal tissues measured by WB. N: non-tumor, T: tumor. c NLRP7 protein in CRC samples and corresponding adjacent normal tissues confirmed by IHC staining. Scale bars, $100 \mu \mathrm{m}$. d Kaplan-Meier survival analysis of patients with CRC according to NLRP7 expression (low: score $<6$, high: score $\geq 6$ ). Knockdown and overexpression of NLRP7 affected CRC cells proliferation as determined by (e) CCK8 assay, (f) colony formation ability and (g) cell apoptosis. The results of transwell assay of the (h) migration and (i) invasion for NLRP7 knockdown and overexpression CRC cells. Scale bars, $100 \mu \mathrm{m}$. ${ }^{* * *} P<$ $0.001,{ }^{*} P<0.01$, and ${ }^{*} P<0.05$. NS: no significance

\section{Results}

NLRP7 protein with high expression level in CRC promotes tumor cell proliferation and metastasis

NLRP7 mRNA levels did not differ significantly between 30 CRC tissues and normal colorectal mucosa by qRTPCR (Fig. 1a), and was confirmed by analysis of gene expression from the GEO dataset (GSE23878, GSE32323, GSE110223, GSE156355) (Additional file 3: Fig. S1A). However, the protein levels of NLRP7 were significantly higher in human CRC tissues than in paired normal tissues as determined by western blotting (Fig. 1b) and confirmed by IHC staining in samples from 115 CRC patients compared with the corresponding adjacent normal tissues (ANTs) (Fig. 1c). The protein expression of NLRP7 in the CRC cohort was significantly correlated with distant metastasis $(P=0.004)$ and advanced clinical stage $(P=0.033)$ (Additional file 1 : Table $S 4)$. Moreover, Kaplan-Meier survival curve analysis demonstrated that CRC patients with increased NLRP7 protein expression had poorer overall five-year survival (Fig. 1d).

Next, we investigated the biological role of NLRP7 in CRC using cell functional assays. Knockdown of NLRP7 significantly decreased DLD-1 cell proliferation compared with that in the negative control, whereas NLRP7 overexpression significantly increased SW480 cell proliferation (Fig. 1e and f, Additional file 3: Fig. S1B). In addition, the proportion of apoptotic cells decreased in SW480 cells overexpressing NLRP7 and increased in DLD-1 cells after knockdown of NLRP7 (Fig. 1g, Additional file 3: Fig. S1C). To determine the role of NLRP7 in CRC metastasis, we performed migration and invasion assays in vitro. The results showed that overexpression of NLRP7 promoted the migration and invasion of SW480 and HT29 cells, whereas NLRP7 knockdown in DLD-1 and HCT116 cells had the opposite effects (Fig. 1h and i). Collectively, these results indicated that upregulated NLRP7 protein in CRC promotes tumor cell proliferation and metastasis.

\section{USP10 interacts with NLRP7 and is highly expressed in CRC}

To identify candidate proteins that interact with NLRP7, a protein pull-down assay was performed. One of the most abundant and specific bands was separated and identified as ubiquitin-specific peptidase 10 (USP10) by liquid chromatography-mass spectrometry (LC/MS-MS) analysis (Fig. 2a), and all proteins that may physically interact with NLRP7 are listed in Additional file 4. IP assays confirmed that USP10 existed in complexes precipitated with antibody against Flag-NLRP7 compared with control IgG (Fig. 2b, upper). Binding of endogenous USP10 to NLRP7 was validated by IP assay with antibody against USP10 (Fig. 2b, lower). IF staining showed that NLRP7 extensively colocalized with USP10 in the cytoplasm (Fig. 2c).

To evaluate the expression profile of USP10 in CRC, we analyzed the GEO database (GSE18105, GSE22598, GSE100179, GSE106582), and the results showed that USP10 mRNA was significantly upregulated in CRC (Fig. 2d). USP10 protein levels were increased in representative CRC patient tissues compared with normal tissues (Fig. 2e). To investigate the function of USP10 in CRC, we performed IHC staining for USP10 using archived CRC tissues as described previously. The results showed that the expression of USP10 was elevated and significantly positively correlated with NLRP7 expression in CRC tissues (Fig. 2f-g).

\section{Degradation of NLRP7 is repressed by USP10 deubiquitination}

To confirm the positive role of USP10 in the regulation of NLRP7, we investigated the effect of changes in USP10 expression on NLRP7 in HCT116 cells. USP10 upregulation increased NLRP7 protein levels (Fig. 3a). However, USP10 had no significant effect on the mRNA levels of NLRP7 in CRC cells (Fig. 3b). Considering that USP10 is a deubiquitinase that removes ubiquitin from its substrates [28], we analyzed the effects of USP10 on the deubiquitination and degradation of NLRP7. Consistently, knockdown of USP10 in HCT116 cells significantly shortened the half-life of the NLRP7 protein in the presence of the protein synthesis inhibitor cycloheximide $(\mathrm{CHX})$ compared with that under control conditions (Fig. 3c). These results suggest that NLRP7 levels are modulated post-translationally through its degradation. Co-IP experiments showed that USP10 knockdown increased the ubiquitination of NLRP7 in HCT116 cells (Fig. 3d).

Next, we identified the lysine on NLRP7 that binds to the Ub moiety in CRC cells. Human NLRP7 truncation 


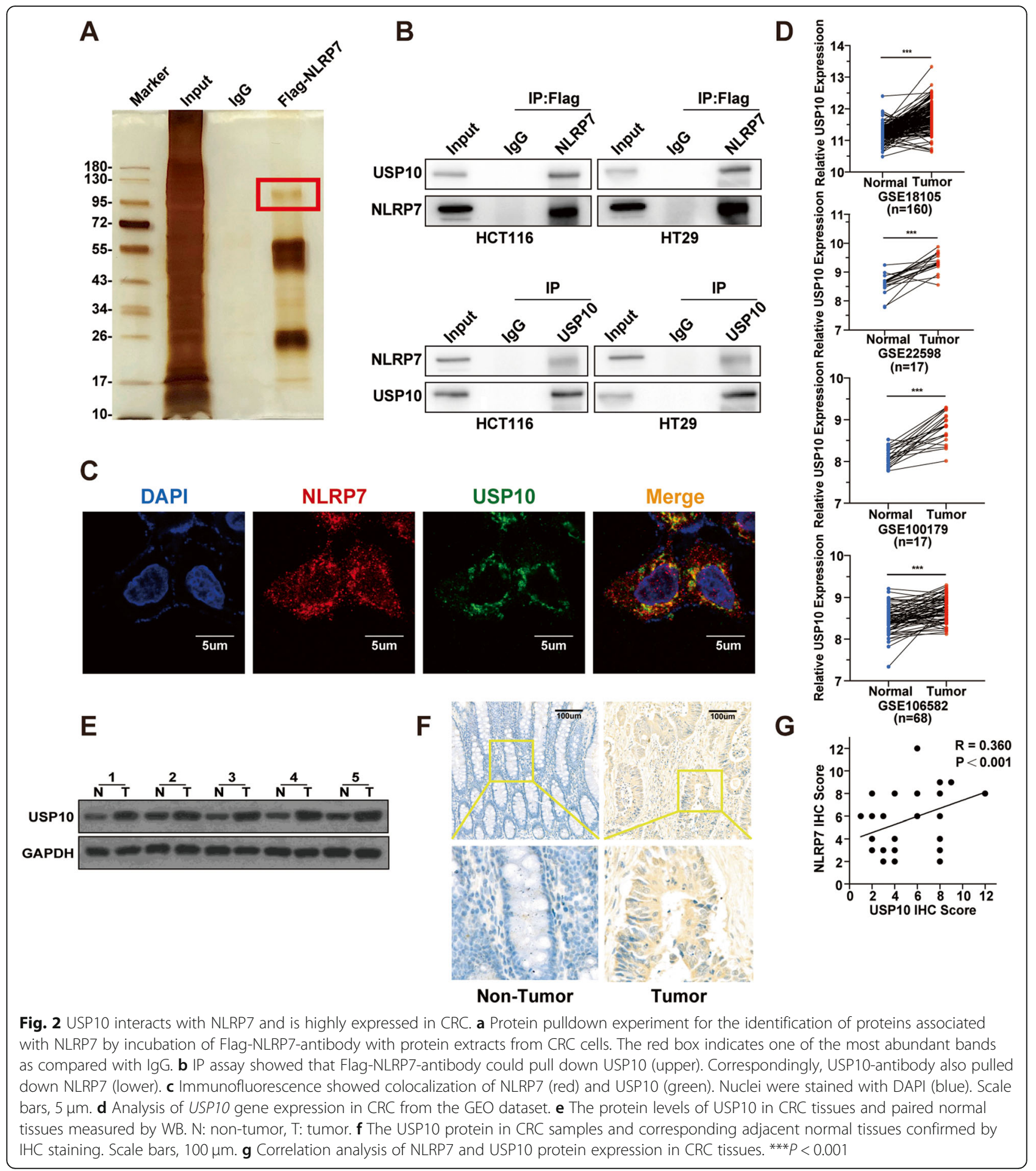

mutants were cloned into the hU6-MCS-CBh-gcGFPIRES-puromycin vector, and the protein stability of ectopically expressed mutants was assessed in the presence of the protein synthesis inhibitor CHX. A significant increase in protein stability was only observed in the 500C NLRP7 mutant (Fig. 3e-g), suggesting that the NLRP7 ubiquitin acceptor site resides within 10 lysine residues between aa 250 and 500. Point mutations were then generated by substituting arginine for lysine at each candidate acceptor site. The protein stability of the K379R mutant increased significantly in cells treated with CHX (Fig. 3h-i). Co-IP studies confirmed that ubiquitination was decreased in K379R NLRP7 compared with that in wild-type NLRP7 (Fig. 3j). These results 


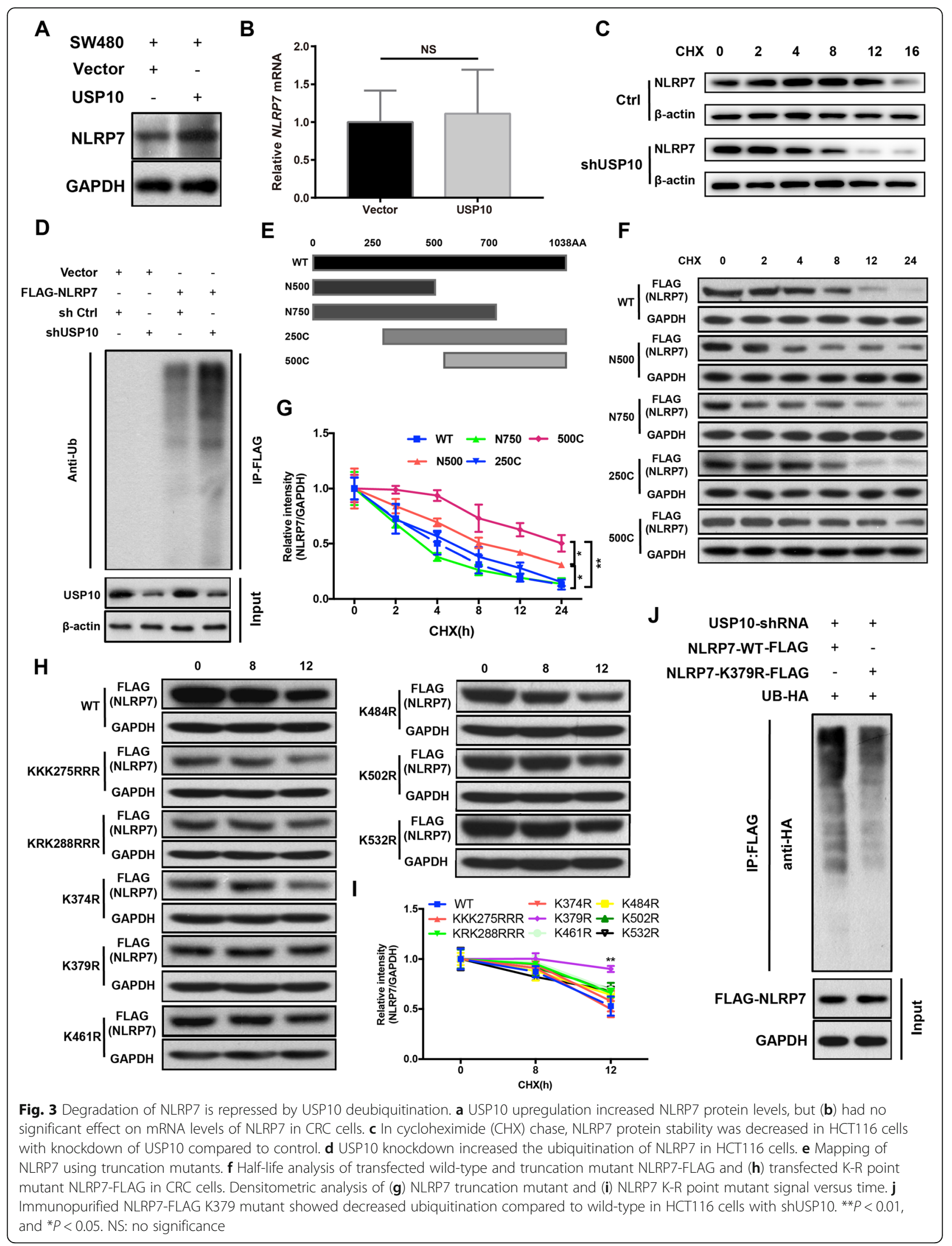




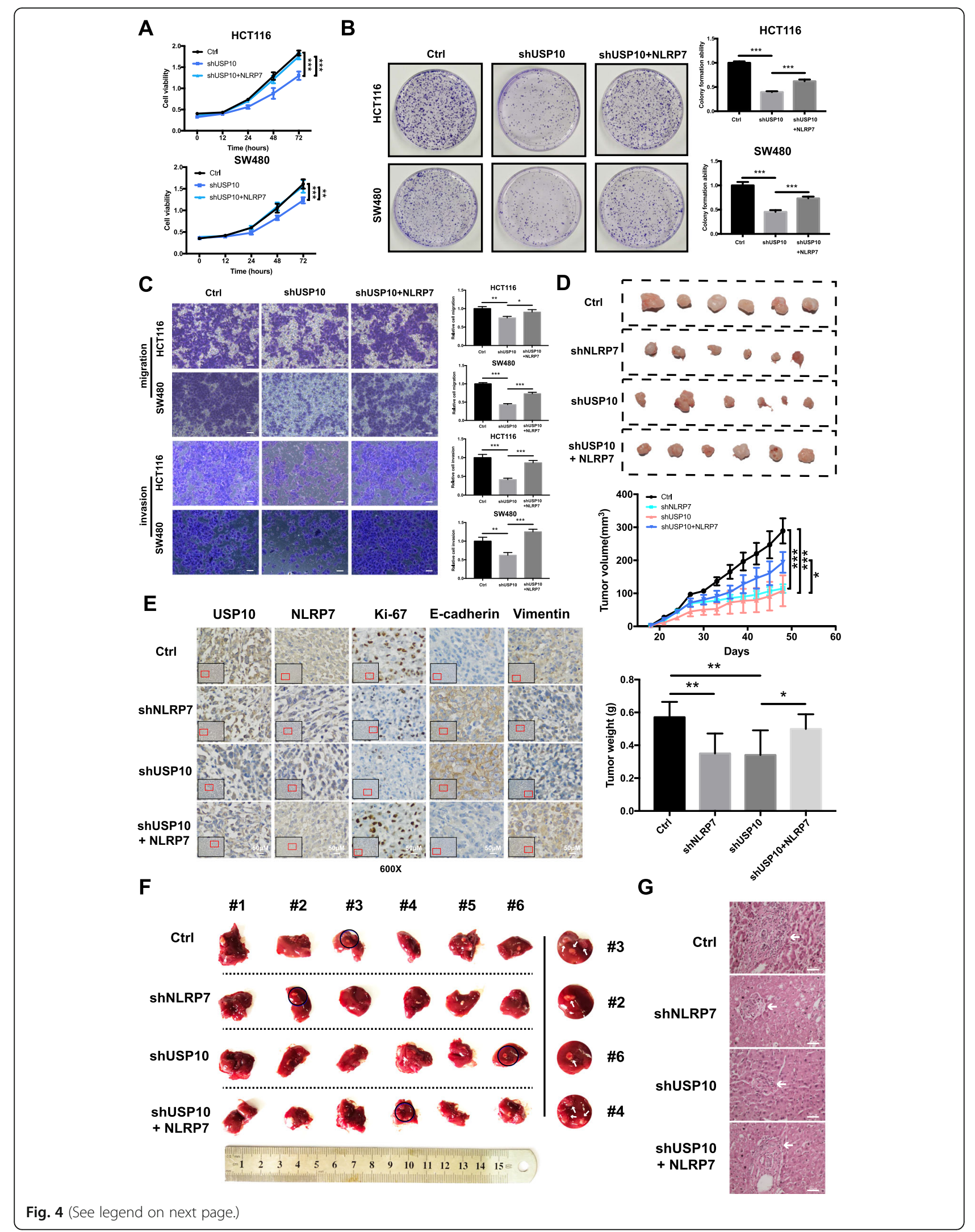


(See figure on previous page.)

Fig. 4 USP10 accelerates the CRC malignant process by upregulating NLRP7. a-c NLRP7 overexpression rescued impaired proliferation, colony formation, migration, and invasive capability caused by USP10 knockdown in HCT116 and SW480 cells. Scale bars, 100 Hm. d NLRP7 or USP10 knockdown inhibited the growth of HCT116 tumors in vivo, whereas overexpression of NLRP7 in USP10 knockdown cells had a synergistic effect on tumor growth. e Representative IHC images of Ki-67, E-cadherin, and vimentin in different xenograft sections (magnification: $\times 600)$. Scale bars, $50 \mu \mathrm{m}$. $\mathbf{f}$ Visible metastatic lesions in the liver, and NLRP7 overexpression reversed the inhibition of liver metastasis by USP10 knockdown. $\mathbf{g}$ Representative images of hematoxylin-eosin staining sections from metastatic nodules in the liver (magnification: $\times 200$ ). Scale bars, $25 \mu \mathrm{m}$. ${ }^{*} * P<0.001,{ }^{* *} P<0.01$, and ${ }^{*} P<0.05$

suggest that K379 is an important lysine acceptor site involved in modulating NLRP7 half-life in CRC cells.

\section{USP10 accelerates the CRC malignant process by upregulating NLRP7}

To explore the role of NLRP7 in the tumor-promoting function of USP10, the effect of NLRP7 overexpression on the tumorigenic potential of USP10-shRNA lentivirusstable-transfected HCT116 and SW480 cells was examined. Downregulation of USP10 expression significantly suppressed the proliferative, migration, and invasive capabilities of HCT116 and SW480 cells, whereas overexpression of NLRP7 attenuated the inhibitory effect of USP10 silencing (Fig. 4a-c).

Tumor xenograft experiments showed that NLRP7 or USP10 knockdown inhibited the growth of HCT116 tumors in vivo, whereas overexpression of NLRP7 in USP10 knockdown cells had a synergistic effect on tumor growth (Fig. 4d). IHC staining for Ki-67, Ecadherin, and vimentin in xenograft tumors was performed and signals were captured under a microscope (Fig. 4e). NLRP7 or USP10 knockdown dramatically inhibited liver metastasis in a mouse model. However, NLRP7 overexpression reversed the inhibition of liver metastasis by USP10 knockdown (Fig. 4f-g). Collectively, the in vitro and in vivo results suggest that NLRP7 is involved in the effect of USP10 on promoting tumor progression and metastasis in CRC.

\section{NLRP7 promotes CCL2 expression and TAM polarization}

To elucidate the molecular mechanism by which NLRP7 promotes CRC progression, CRC cells with stable NLRP7 overexpression and knockdown were subjected to RNA sequencing (RNA-seq). RNA-seq identified 3065 transcripts that were significantly upregulated by NLRP7 overexpression (fold change $>2$ ) and 2389 transcripts that were significantly downregulated (fold change $<0.5$ ) by NLRP7 knockdown (Fig. 5a). The results showed that 398 genes were positively correlated with NLRP7 expression and overlapped between the two groups. The 398 transcripts are listed in Additional file 5, and the GO and KEGG enrichment analysis results are shown in Additional file 3: Fig. S2A and S2B. Cytokines and chemokines, as downstream signal transduction molecules, are essential for NLR family members to ensure their function and influence disease outcomes [6, 10, 29, 30]. Therefore, we decided to focus on cytokines and chemokines that are regulated by NLRP7 in cancer cells. Among 398 transcripts, four cytokines/chemokines, including CCL2, TNFSF13, CXCL8, and TNFSF15, were positively correlated with the expression of NLRP7. Among them, CCL2 mRNA detected by qRT-PCR was dramatically increased in NLRP7-overexpressing cells and decreased in NLRP7 knockdown cells (Fig. 5b). ELISA with a specific anti-CCL2 antibody confirmed that CCL2 secretion was induced by NLRP7 overexpression and significantly repressed by NLRP7 knockdown (Fig. 5c). Because high levels of CCL2 are correlated with increased numbers of TAMs in tumor tissues in many human tumors [31], we speculated that the recruitment of TAMs by CCL2 is involved in the function of NLRP7 in promoting CRC progression.

To test the hypothesis that NLRP7 regulates the release of chemokines by CRC cells to modulate TAM polarization, THP-1 cells were incubated with conditioned medium (CM) collected from different CRC cell lines, and the expression of TAM polarization markers was analyzed. The culture medium from NLRP7 overexpressing cells promoted the chemotaxis of THP-1 human monocytes (Additional file 3: Fig. S3A). Moreover, macrophages (F4/80) were reduced in the tumor microenvironment (TME) of xenograft tumor tissues in NLRP7 knockdown group compared with control, as detected by immunohistostaining (Fig. 5d). The CM from NLRP7 overexpressing CRC cells, but not the control medium, upregulated the mRNA expression of $V E G F$ and $A R G 1$ which were used as indicators for the functional polarization of TAMs promoted by tumor signals [32] (Fig. 5e). Flow cytometry showed that the CD68(+)/CD206(+) subpopulations were more abundant, whereas CD68(+)/CD86(+) subpopulations were less abundant in cells overexpressing NLRP7 than in the controls (Fig. 5f). The opposite effect was observed in THP-1 cells incubated with CM from NLRP7 knockdown cells.

\section{CCL2 secretion from CRC cells is necessary for NLRP7 inducing TAM polarization}

To investigate whether the effect of CRC cells on TAM polarization is mediated by the secretion of CCL2, 


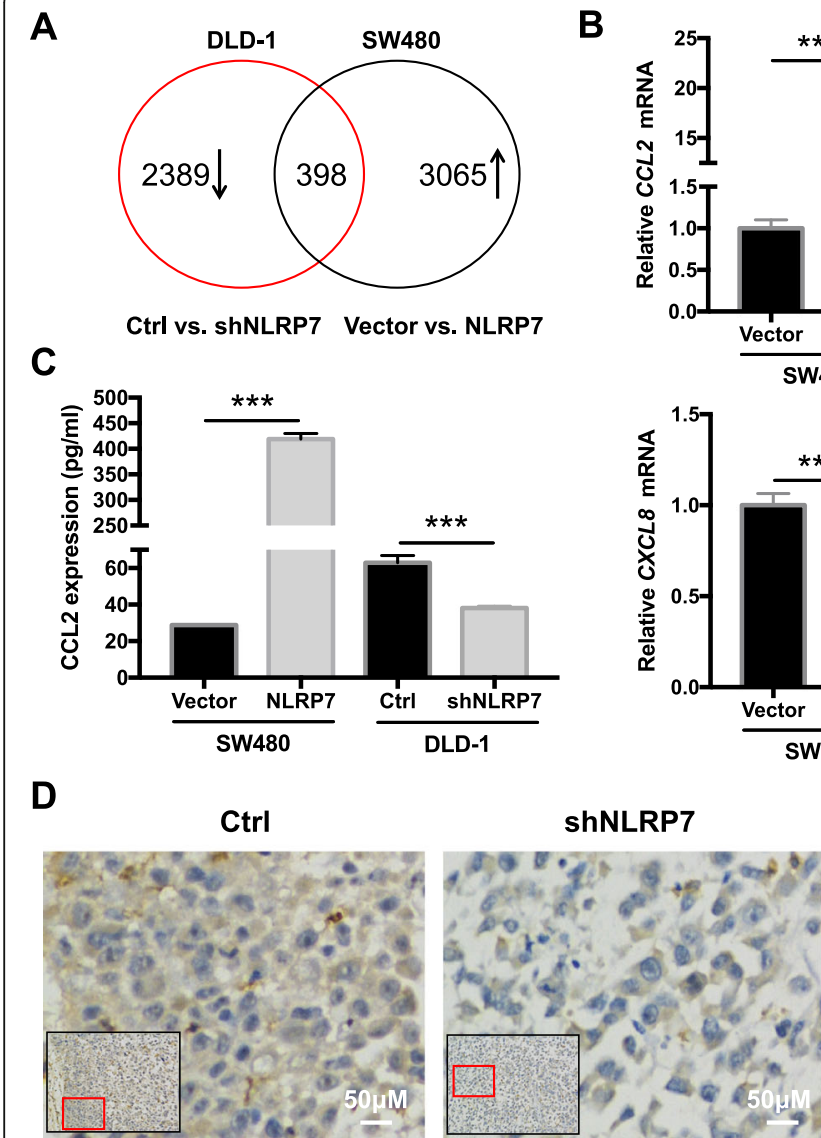

F4/80 (600X)

F

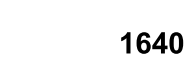

1640

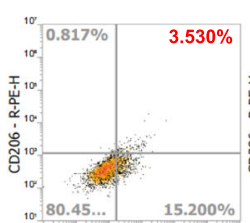

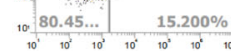

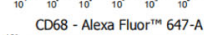

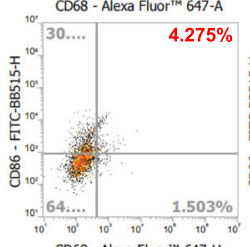

CD68 - Alexa Fluorm $647-\mathrm{H}$
DLD-1

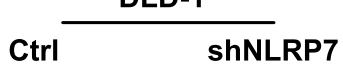

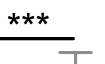
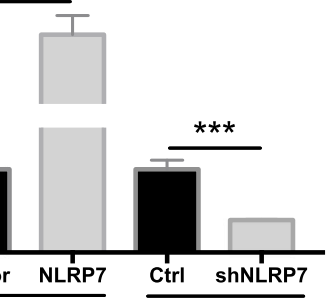

W480
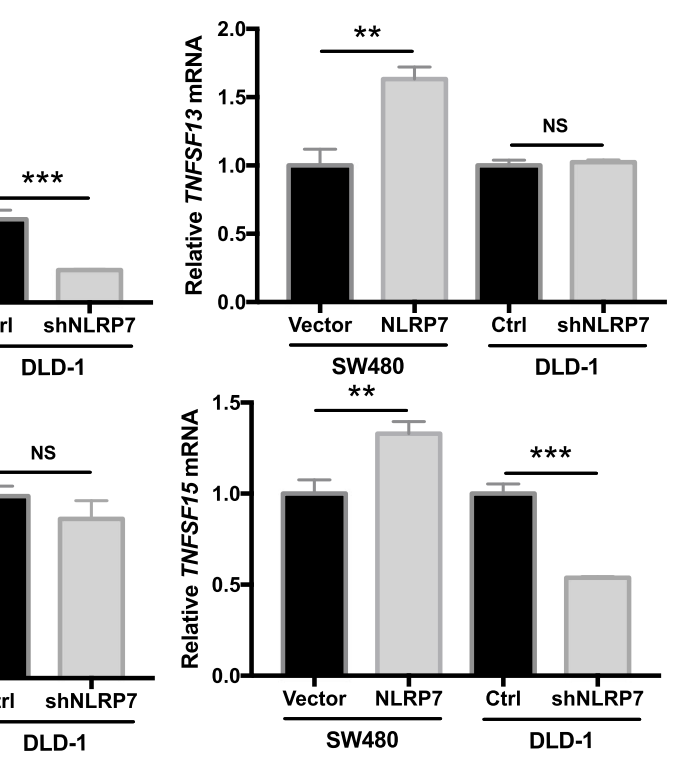

E
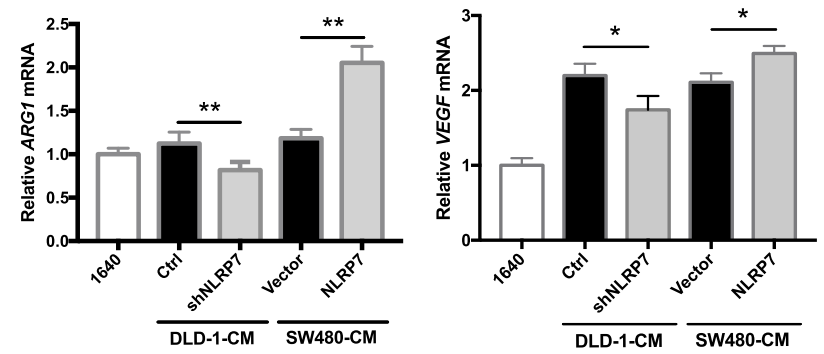

SW480
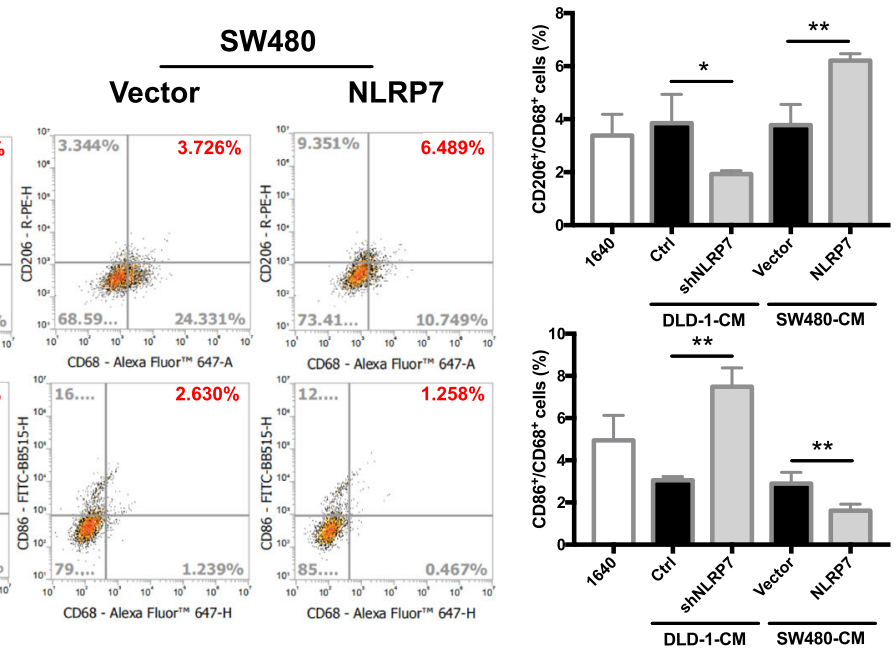

Fig. 5 NLRP7 promotes CCL2 expression and TAM polarization. a Number of altered transcripts detected in CRC cells with either NLRP7 overexpression or knockdown. b qRT-PCR verification of transcription of four cytokines/chemokines, including CCL2, TNFSF13, CXCL8, and TNFS F15, in CRC cells with NLRP7 overexpression or knockdown. c ELISA confirmed CCL2 secretion levels in CRC cells with NLRP7 overexpression or knockdown. d Macrophages detected by immunohistostaining of the xenograft tumor tissues from NLRP7 knockdown and control groups (magnification: $\times 600$ ). Scale bars, $50 \mu \mathrm{m}$. e Expression levels of M2-like macrophage marker changes after treatment with culture medium from NLRP7 overexpressing or knockdown CRC cells. f Flow cytometry was used to evaluate CD68(+)/CD206(+) and CD68(+)/CD86(+) subpopulations. *** $P<0.001,{ }^{* *} P<0.01$, and ${ }^{*} P<0.05$. NS: no significance 
A

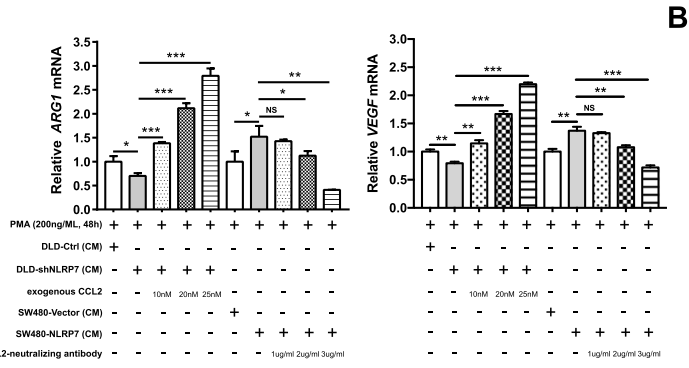

C

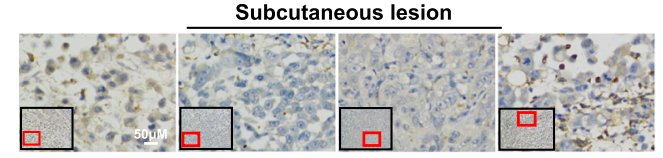

Liver metastatic lesion

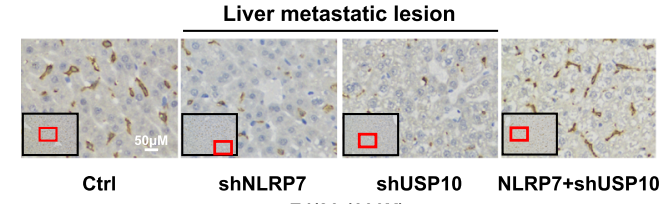
$\mathrm{F} 4 / 80(600 \mathrm{X})$

D
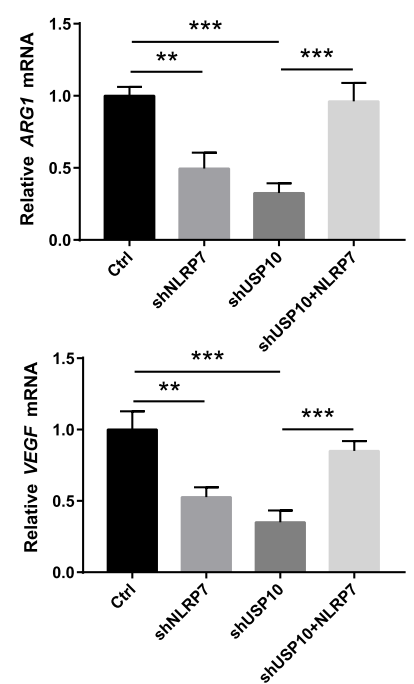

E
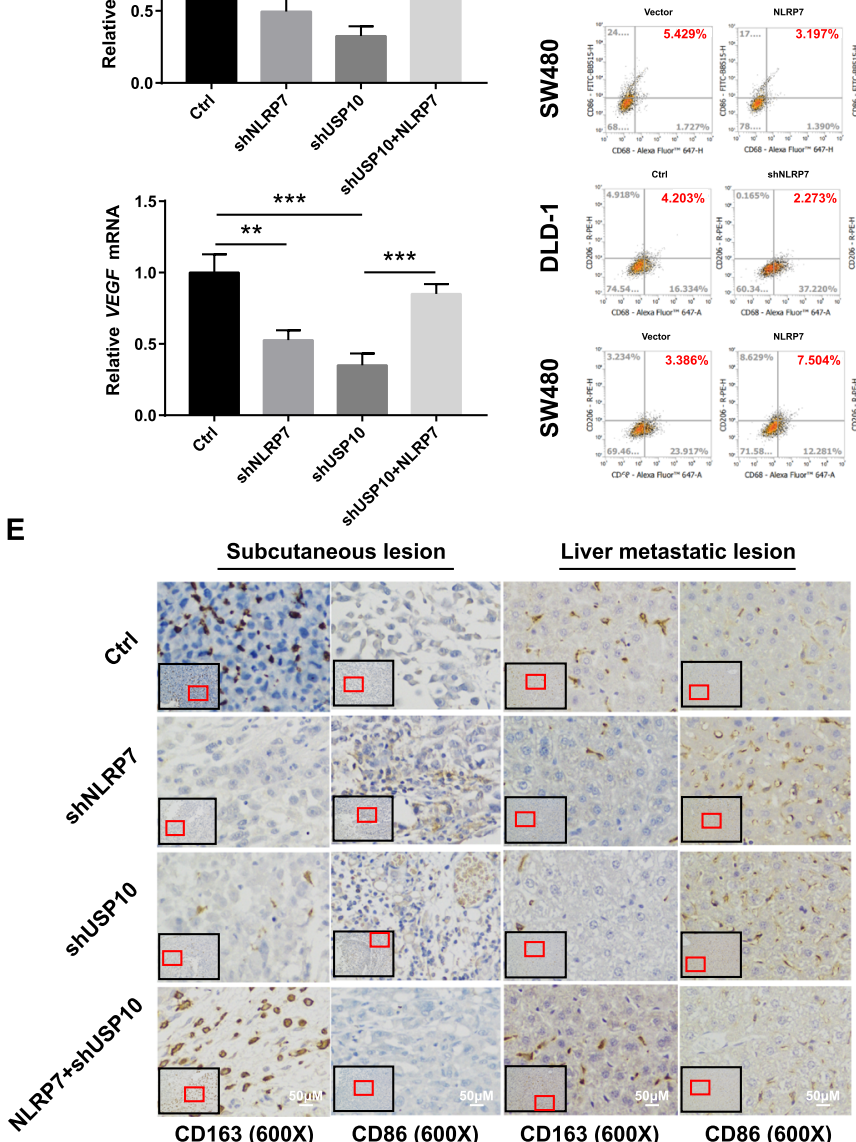

B
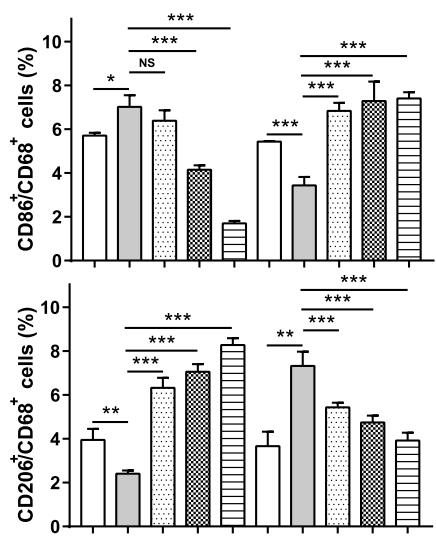

PMA (200ng/ML, 48h) ++++++++++

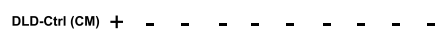

DLD-shNLRP7 (CM) $-++++-\quad-\quad-$

exogenous CCL2 - - 10nM 20nM 25nM - - - -

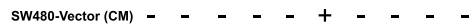

SW480-NLRP7 (CM) - $\quad-\quad-\quad-\quad+\quad+++$

CCL2-neutralizing antibody - - - - - - - $1 \mathrm{ug} / \mathrm{m} / 2 \mathrm{ug} / \mathrm{m} / \mathrm{3ug} / \mathrm{ml}$
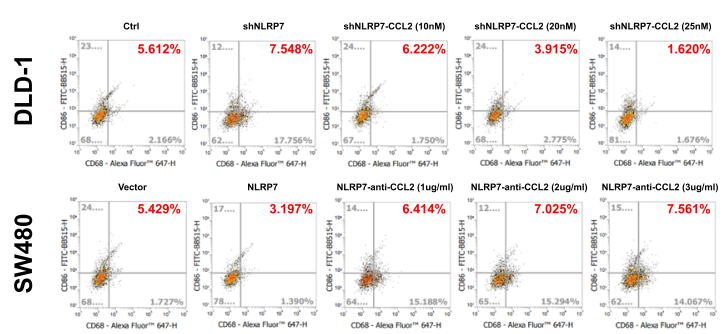

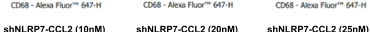

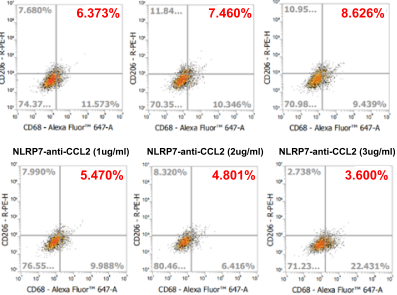

$\mathbf{F}$
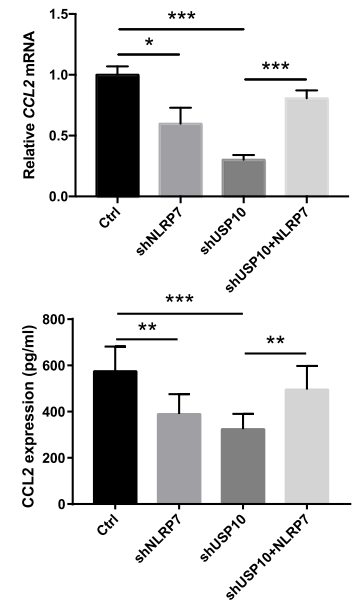

Fig. 6 (See legend on next page.) 
(See figure on previous page.)

Fig. 6 CCL2 secretion from CRC cells is necessary for NLRP7 induction of TAM polarization. a-b The results of qPCR and flow cytometry confirmed that recombinant CCL2 rescued the M2-like macrophage polarization effect from CM of NLRP7 knockdown cells, and CCL2-neutralizing antibody suppressed M2-like macrophage polarization effect from CM of NLRP7 overexpressing cells. c Macrophages in xenograft tumor tissues from control, NLRP7 knockdown, USP10 knockdown, and USP10 knockdown with NLRP7 overexpression (magnification: $\times 600$ ). Scale bars, $50 \mu$ m. d Expression level of TAM functional polarization markers in xenograft tumor tissues. e CD86-positive M1 macrophages and CD163-positive M2 macrophages in xenograft tumor tissues from four groups (magnification: $\times 600$ ). Scale bars, $50 \mu \mathrm{m}$. $\mathbf{f}$ The expression levels of CCL2 in peripheral blood and tumor tissues of animals from four groups. ${ }^{* *} P<0.001$, ${ }^{* *} P<0.01$, and ${ }^{*} P<0.05$. NS: no significance

recombinant CCL2 and a CCL2-neutralizing antibody were added to the CM from NLRP7 knockdown cells and the CM from NLRP7 overexpressing cells, respectively. The results of qPCR and flow cytometry confirmed that CCL2 induced M2-like macrophage polarization in a dose-dependent manner (Fig. 6a-b). Moreover, the CM from SW480 cells treated with CCL2-neutralizing antibody significantly inhibited the chemotaxis of THP-1 cells, which was restored by exogenous CCL2 addition (Additional file 3: Fig. S3B).

In animal studies, macrophages were reduced in the TME of xenograft tumor tissues in NLRP7 and USP10 knockdown groups and were increased in the NLRP7 overexpressing group (Fig. 6c). The Vegf and Arg1 mRNA expression in tissues was higher in NLRP7overexpressing tumors and lower in sh-NLRP7 and shUSP10 tumors than in the control group (Fig. 6d). Furthermore, IHC staining for the M1 marker CD86 showed that accumulation of CD86-positive M1 macrophages was greater in the stroma of sh-NLRP7 tumors and sh-USP10 tumors than in that of scrambled shRNA treated tumors, and NLRP7 overexpression suppressed the accumulation of CD86-positive M1 macrophages. CD163-positive M2 macrophages were more abundant in NLRP7-overexpressing tumors and less abundant in sh-NLRP7 tumors and sh-USP10 tumors than in the control tumors (Fig. 6e). The expression levels of CCL2 in peripheral blood and tumor tissues were significantly lower in sh-NLRP7-treated and sh-USP10-treated mice than in control mice and higher in NLRP7-treated mice with USP10 knockdown (Fig. 6f). Thus, data from cells and animal models suggested that NLRP7 enhances TAM polarization by promoting CCL2 secretion from CRC cells.

\section{NLRP7 facilitates transactivation of CCL2 via NF-KB}

Considering that CCL2 is a classical NF- $\mathrm{kB}$ target gene [25], we next examined whether the effect of NLRP7 on CCL2 expression and monocyte chemotaxis is mediated by enhanced NF-kB signaling. The NF- $\mathrm{kB}$ circuit involves p65 phosphorylation and nuclear translocation, which lead to gene activation. Phosphorylation of p65 was increased in NLRP7-overexpressing cells, whereas the protein levels did not change (Fig. 7a). IF analysis demonstrated that NLRP7 overexpression increased p65
NF- $\mathrm{kB}$ accumulation in the nucleus compared with that in control cells (Fig. 7b). These results suggest that NLRP7 promotes p65 NF-kB accumulation in the nucleus by enhancing its phosphorylation.

Next, we determined the effect of NLRP7 on CCL2 expression in response to NF- $\mathrm{KB}$ activation. We confirmed that the NLRP7-induced expression of CCL2 was significantly suppressed following NF- $\mathrm{KB}$ inhibition using the NF- $\kappa$ B-specific inhibitor CAPE (Fig. 7c). Luciferase reporter gene analysis showed that ectopic expression of NLRP7 induced, whereas CAPE suppressed CCL2 transcription (Fig. 7d). These results suggest that NLRP7 regulates the expression of CCL2 by modulating the NF$\kappa \mathrm{B}$ signaling pathway.

Collectively, the present results indicated that NLRP7 in CRC cells interacts with and is deubiquitinated by USP10, resulting in increased NLRP7 protein stability (Fig. 7e). NLRP7 promoted the secretion of CCL2 by activating the NF- $\mathrm{KB}$ signaling pathway. CCL2 secreted by CRC cells promoted the polarization of pro-tumor M2like macrophages. Therefore, upregulation of NLRP7 in CRC facilitated tumor cell proliferation and metastasis.

\section{Discussion}

NLRs affect multiple stages of cancer progression in several malignancies, including colorectal cancer, gastric cancer, hepatocellular carcinoma, and other cancers [6]. The exact roles of NLR family members in CRC are diverse and complex. In this study, we showed that NLRP7 is upregulated at the protein level in CRC samples compared with matched non-tumor controls, and that overexpression of NLRP7 is associated with poor prognosis of CRC patients. Functional studies in vitro and in vivo revealed that NLRP7 promoted tumor cell proliferation and metastasis. Previous studies focused on the role of NLRP7 during embryonic development [33]. The present results thus expand our knowledge of the role of this protein in cancer.

At the beginning of this study, we observed an increase in NLRP7 protein levels without changes in mRNA levels in CRC tissues compared with levels in the corresponding ANTs. This phenomenon needed to be explained mechanistically. Protein ubiquitination is an important post-translational modification that regulates the degradation of proteins, but not alteration 


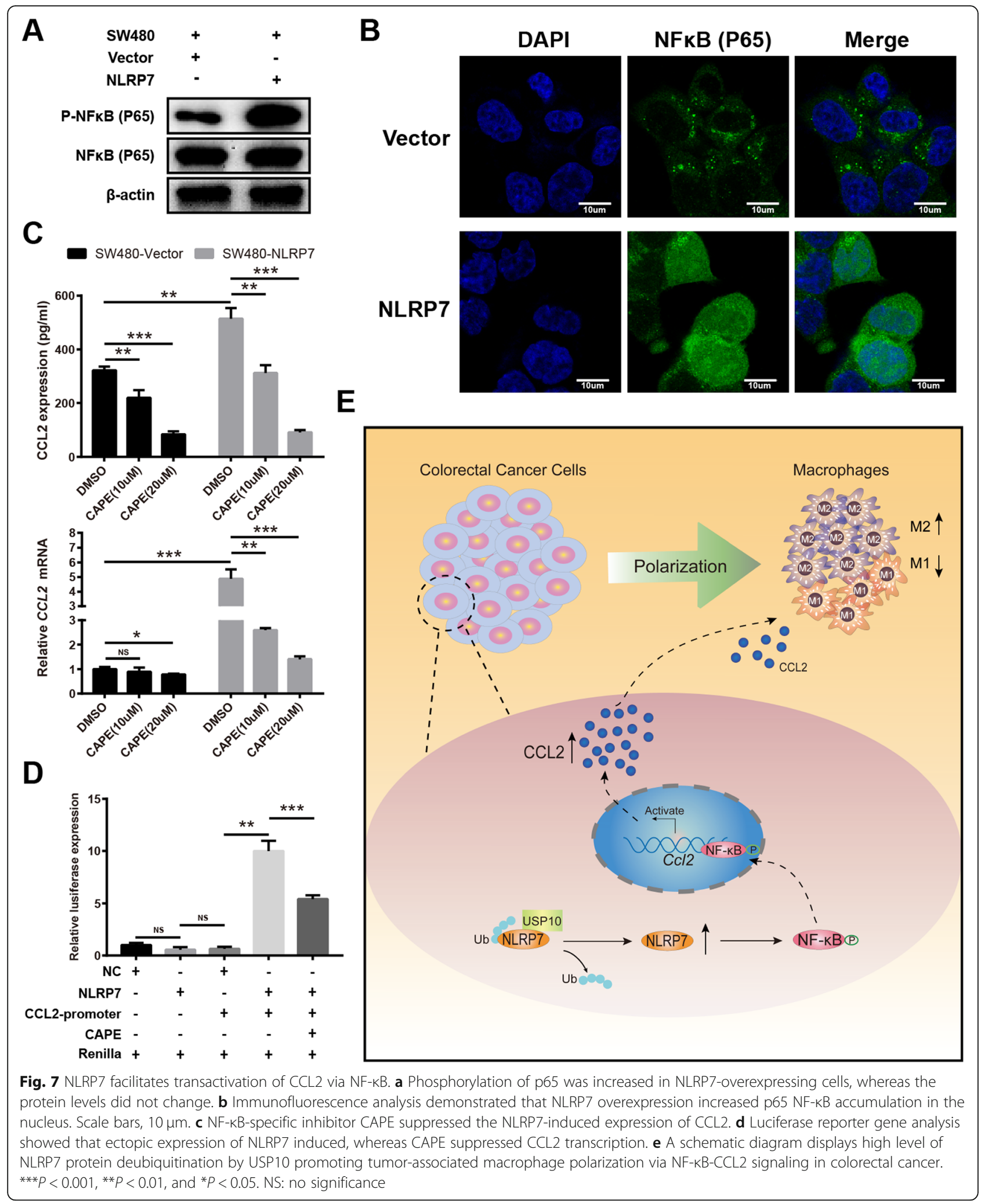

of mRNA [34]. A recent study showed that STAMbinding protein (STAMBP), a deubiquitinating enzyme, modulates NLRP7 protein stability by catalyzing its deubiquitination to increase NLRP7 abundance [35]. Moreover, some other NLR family members, including NLRP3 and NOD2, were also demonstrated to 
be affected by de-ubiquitinase enzymes (DUBs) [3639]. Even without experimental validation, the above literature suggests that NLRP7 regulated by deubiquitination is a well-founded hypothesis. Here, we identified USP10 as a critical regulator of NLRP7 deubiquitination and stabilization in CRC cells. We identified K379 as an important lysine acceptor site that mediates NLRP7 ubiquitination in CRC cells. However, a previous study identified KRK288/290 as the main lysine acceptor sites affecting ubiquitination of NLRP7 [35]. This discrepancy may be related to differences in the cells and conditions used for analyzing NLRP7, as well as different biological functions of NLRP7.

Several lines of evidence support the diverse roles of USP10 in tumorigenesis in different cancer types. For example, in hepatocellular carcinoma, Zhu et al. reported that USP10 promotes proliferation [40], whereas Lu et al. found that USP10 suppresses tumor progression by inhibiting mTOR activation [41]. In the present study, USP10 was overexpressed in CRC tissues, as determined by our data and public GEO database information. Targeting USP10 by shRNA inhibited CRC proliferation and metastasis, and this effect was reversed by overexpression of NLRP7. This result indicates that NLRP7 may be a key effector molecule for the cancer-promoting effects of USP10 in CRC cells.

TAMs constitute a plastic and heterogeneous cell population of the tumor microenvironment, and they are closely related to cancer progression and resistance to therapy [42]. Here, we found that NLRP7 overexpression in CRC cells enhanced TAM polarization, which may be one of the mechanisms by which NLRP7 promotes CRC progression. Polarization of TAMs is regulated by multiple microenvironmental cytokines, growth factors, epigenetic regulators, and other signals derived from tumor and stromal cells [43]. We showed that NLRP7 promoted the transcription of CCL2, and the secretion of CCL2 was increased in NLRP7 overexpressing CRC cells. Several studies reported that CCL2 not only promotes tumor invasion and metastasis, but also acts as a macrophage recruiter and M2-stimulating factors [44-46]. We showed that M2-like macrophage polarization induced by overexpression of NLRP7 in CRC cells is mediated by CCL2, and this effect can be attenuated by a CCL2-neutralizing antibody in vitro. Moreover, the protein levels of CCL2 in peripheral blood were significantly decreased in sh-NLRP7-treated mice and increased in NLRP7-treated mice, whereas M2-like macrophages were more abundant in NLRP7overexpressing tumors and less abundant in shNLRP7 tumors. These results indicate that high expression of NLRP7 in CRC promotes macrophage polarization in a CCL2-dependent manner.
Mechanistically, we demonstrated that the NF- $\kappa B$ pathway is essential for CCL2 production induced by NLRP7 in CRC cells. We confirmed that NLRP7 promotes the phosphorylation of $\mathrm{p} 65 \mathrm{NF}-\mathrm{kB}$, leading to increased nuclear accumulation of $\mathrm{p} 65 \mathrm{NF}-\mathrm{kB}$. NLRs were previously shown to modulate NF- $\mathrm{kB}$ signaling by inhibition or activation [47]. NLRC1 activates NF- $\mathrm{BB}$ signaling [48], whereas NLRP12 negatively regulates NF- $\mathrm{B}$ [13]. In this study, the translocation of NF- $\mathrm{KB}$ from the cytoplasm to the nucleus promoted by NLRP7 enhanced the binding of NF- $\mathrm{KB}$ to the CCL2 promoter, thereby increasing its expression and secretion. Previous studies showed that the genes encoding CCL2 are downstream targets of the NF- $\mathrm{kB}$ transcription factor, which are crucial for cancer progression and metastasis [25, 49]. Therefore, the NF- $\mathrm{kB}-\mathrm{CCL} 2$ signaling axis plays a critical role in the effect of NLRP7 on promoting TAM polarization and CRC progression.

\section{Conclusion}

In summary, we identified a potential role of NLRP7 in the pathogenesis and metastasis of CRC. NLRP7 protein levels were increased by USP10-mediated deubiquitination in CRC cells, which induced the polarization of pro-tumor M2-like macrophages via NF-kB pathway-mediated CCL2 secretion. These findings provide a better understanding of the oncogenic mechanisms of NLRP7, and may advance the development of therapeutic strategies for CRC.

\section{Abbreviations \\ CRC: Colorectal cancer; TME: Tumor microenvironment; TAMs: Tumor- associated macrophages; CCL2: C-C motif chemokine 2; NLRs: NOD-like receptors; PAMPs: Pathogen-associated molecular patterns; NLRP7: NACHT, LRR and PYD domains-containing protein 7; UC: Ulcerative colitis; IBD: Inflammatory bowel disease; OS: Overall survival; USP10: ubiquitin- specific-processing protease 10; ANTs: Adjacent normal tissues; IHC: Immunohistochemistry; IP: Immunoprecipitation; \\ IF: Immunofluorescence; CHX: Cycloheximide; CM: Conditioned medium}

\section{Supplementary Information}

The online version contains supplementary material available at https://doi. org/10.1186/s13046-021-01920-y.

Additional file 1: Table S1. Primer sequences for real-time PCR. Table S2. The antibodies used in western blot, flow cytometry, and IHC. Table S3. Primer sequences for truncation mutant and site-directed mutagenesis. Table S4. Clinicopathological correlation of high NLRP7 expression in CRC.

Additional file 2: Unprocessed original blots.

Additional file 3: Figure S1. (A) Analysis of NLRP7 gene expression from the GEO dataset. (B) Representative images of colony formation assay. (C) Cell apoptosis in NLRP7 knockdown and overexpression CRC cells manifested by flow cytometry analysis. Figure S2. The GO (A) and KEGG (B) enrichment analysis results of 398 genes positively related with NLRP7 expression. Figure S3. (A) Chemotaxis of THP-1 cells treated with culture medium from cells overexpressing NLRP7. (B) CCL2-neutralizing antibody inhibited THP-1 cell chemotaxis, which was restored by exogenous CCL2.

Additional file 4: The list of proteins physically interacted with NLRP7. 
Additional file 5: The list of 398 genes that were positively correlated with NLRP7 expression in CRC cells.

\section{Acknowledgements}

We would like to acknowledge the reviewers for their helpful comments on this paper.

\section{Authors' contributions}

$\mathrm{YZ}$ and $\mathrm{PZ}$ conceived and designed this study; $\mathrm{BL}, \mathrm{ZQ}, \mathrm{DH}, \mathrm{ZC}$, and JZ performed major experiments; MW, EX, JL, and DS assisted with cell experiments; QS, LY and SC assisted with animal experiments; BL, ZC and JL analyzed and interpreted the data; $\mathrm{BL}, \mathrm{ZQ}, \mathrm{DH}$, and $\mathrm{ZC}$ drafted the manuscript; $Y Z$ and $P Z$ revised the manuscript. The authors read and approved the final manuscript.

\section{Funding}

This work was supported by grants from the National Key R\&D Program of China (grant nos. 2018YFC1315000, 2018YFC1315005), the National Natural Science Foundation of China (grant nos. 81672329, 82002515, 81861168036), the Science and Technology Commission Foundation of Shanghai Municipality (grant nos. 19411951600, 19411951601), the Dawn Program of the Shanghai Education Commission (grant no. 18SG08), and the Shanghai Sailing Program (grant no. 20YF1407200). The funders had no role in the study design, data collection/analysis, decision to publish, or preparation of the manuscript.

\section{Availability of data and materials}

All data in our study will be available upon reasonable request.

\section{Declarations}

\section{Ethics approval and consent to participate}

The present study was approved by the Ethics Committee of Zhongshan Hospital, Fudan University, and was performed according to the Declaration of Helsinki. All patients recruited into the study signed written informed consent forms.

\section{Consent for publication}

All authors read and approved the final manuscript.

\section{Competing interests}

The authors declare that they have no conflicts of interest.

\section{Author details}

${ }^{1}$ Endoscopy Center, Zhongshan Hospital of Fudan University, 180 Fenglin Road, Shanghai 20032, People's Republic of China. 'Endoscopy Research Institute of Fudan University, Shanghai 20032, People's Republic of China. ${ }^{3}$ Endoscopy Center, Xuhui Hospital, Zhongshan Hospital of Fudan University, Shanghai 20031, People's Republic of China. ${ }^{4}$ Department of Gastroenterology, Xuhui Hospital, Zhongshan Hospital of Fudan University, Shanghai 20031, People's Republic of China.

\section{Received: 20 November 2020 Accepted: 21 March 2021}

\section{Published online: 10 April 2021}

\section{References}

1. Bray F, Ferlay J, Soerjomataram I, Siegel RL, Torre LA, Jemal A. Global cancer statistics 2018: GLOBOCAN estimates of incidence and mortality worldwide for 36 cancers in 185 countries. CA Cancer J Clin. 2018;68(6):394-424. https://doi.org/10.3322/caac.21492.

2. Allemani C, Matsuda T, Di Carlo V, Harewood R, Matz M, Niksic M, et al. Global surveillance of trends in cancer survival 2000-14 (CONCORD-3): analysis of individual records for 37513025 patients diagnosed with one of 18 cancers from 322 population-based registries in 71 countries. Lancet. 2018;391(10125):1023-75. https://doi.org/10.1016/S0140-6736(17)33326-3.

3. Xu RH, Muro K, Morita S, Iwasa S, Han SW, Wang W, et al. Modified XELIRI (capecitabine plus irinotecan) versus FOLFIRI (leucovorin, fluorouracil, and irinotecan), both either with or without bevacizumab, as second-line therapy for metastatic colorectal cancer (AXEPT): a multicentre, open-label randomised, non-inferiority, phase 3 trial. Lancet Oncol. 2018;19(5):660-71. https://doi.org/10.1016/S1470-2045(18)30140-2.

4. Wilmanski JM, Petnicki-Ocwieja T, Kobayashi KS. NLR proteins: integral members of innate immunity and mediators of inflammatory diseases. J Leukoc Biol. 2008;83(1):13-30. https://doi.org/10.1189/jlb.0607402.

5. Grivennikov SI, Greten FR, Karin M. Immunity, inflammation, and cancer. Cell. 2010;140(6):883-99. https://doi.org/10.1016/j.cell.2010.01.025.

6. Liu P, Lu Z, Liu L, Li R, Liang Z, Shen $M$, et al. NOD-like receptor signaling in inflammation-associated cancers: from functions to targeted therapies. Phytomedicine. 2019;64:152925. https://doi.org/10.1016/j.phymed.2019.1 52925

7. Drexler SK, Bonsignore L, Masin M, Tardivel A, Jackstadt R, Hermeking $H_{\text {, }}$ et al. Tissue-specific opposing functions of the inflammasome adaptor ASC in the regulation of epithelial skin carcinogenesis. Proc Natl Acad Sci U S A. 2012;109(45):18384-9. https://doi.org/10.1073/pnas.1209171109.

8. Hu B, Elinav E, Huber S, Strowig T, Hao L, Hafemann A, et al. Microbiotainduced activation of epithelial IL-6 signaling links inflammasome-driven inflammation with transmissible cancer. Proc Natl Acad Sci U S A. 2013; 110(24):9862-7. https://doi.org/10.1073/pnas.1307575110.

9. Bauer C, Duewell P, Mayer C, Lehr HA, Fitzgerald KA, Dauer M, et al. Colitis induced in mice with dextran sulfate sodium (DSS) is mediated by the NLRP3 inflammasome. Gut. 2010;59(9):1192-9. https://doi.org/10.1136/gut.2 009.197822

10. Saxena M, Yeretssian G. NOD-like receptors: master regulators of inflammation and Cancer. Front Immunol. 2014:5:327.

11. Evavold CL, Kagan JC. Inflammasomes: threat-assessment organelles of the innate immune system. Immunity. 2019;51(4):609-24. https://doi.org/10.101 6/j.immuni.2019.08.005.

12. Man SM. Inflammasomes in the gastrointestinal tract: infection, cancer and gut microbiota homeostasis. Nat Rev Gastroenterol Hepatol. 2018;15(12): 721-37. https://doi.org/10.1038/s41575-018-0054-1.

13. Allen IC, Wilson JE, Schneider M, Lich JD, Roberts RA, Arthur JC, et al. NLRP12 suppresses colon inflammation and tumorigenesis through the negative regulation of noncanonical NF-kappaB signaling. Immunity. 2012 36(5):742-54. https://doi.org/10.1016/j.immuni.2012.03.012.

14. Zaki MH, Vogel P, Malireddi RK, Body-Malapel M, Anand PK, Bertin J, et al. The NOD-like receptor NLRP12 attenuates colon inflammation and tumorigenesis. Cancer Cell. 2011;20(5):649-60. https://doi.org/10.1016/j.ccr.2 011.10.022.

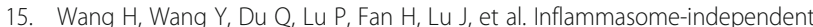
NLRP3 is required for epithelial-mesenchymal transition in colon cancer cells. Exp Cell Res. 2016;342(2):184-92. https://doi.org/10.1016/j.yexcr.2016. 03.009.

16. Dupaul-Chicoine J, Arabzadeh A, Dagenais M, Douglas T, Champagne C, Morizot A, et al. The Nlrp3 Inflammasome suppresses colorectal Cancer metastatic growth in the liver by promoting natural killer cell Tumoricidal activity. Immunity. 2015;43(4):751-63. https://doi.org/10.1016/j.immuni.2015. 08.013

17. Mahadevan S, Wen S, Wan YW, Peng HH, Otta S, Liu Z, et al. NLRP7 affects trophoblast lineage differentiation, binds to overexpressed $Y Y 1$ and alters CpG methylation. Hum Mol Genet. 2014;23(3):706-16. https://doi.org/10.1 093/hmg/ddt457.

18. Murdoch S, Djuric U, Mazhar B, Seoud M, Khan R, Kuick R, et al. Mutations in NALP7 cause recurrent hydatidiform moles and reproductive wastage in humans. Nat Genet. 2006:38(3):300-2. https://doi.org/10.1038/ng1740.

19. Onoufriadis A, Stone K, Katsiamides A, Amar A, Omar Y, de Lange KM, et al. Exome sequencing and genotyping identify a rare variant in NLRP7 gene associated with ulcerative colitis. J Crohns Colitis. 2018;12(3):321-6. https:// doi.org/10.1093/ecco-jcc/jjjx157.

20. Davis BK, Philipson C, Hontecillas R, Eden K, Bassaganya-Riera J, Allen IC. Emerging significance of NLRs in inflammatory bowel disease. Inflamm Bowel Dis. 2014;20(12):2412-32. https://doi.org/10.1097/MIB. 0000000000000151.

21. Olafsson S, Mclntyre RE, Coorens T, Butler T, Jung H, Robinson PS, et al. Somatic evolution in non-neoplastic IBD-affected Colon. Cell. 2020;182(3): 672-84 e11. https://doi.org/10.1016/j.cell.2020.06.036.

22. Deng Q, Geng Y, Zhao L, Li R, Zhang Z, Li K, et al. NLRP3 inflammasomes in macrophages drive colorectal cancer metastasis to the liver. Cancer Lett. 2019;442:21-30. https://doi.org/10.1016/j.canlet.2018.10.030.

23. Weiser MR. AJCC 8th edition: colorectal Cancer. Ann Surg Oncol. 2018;25(6): 1454-5. https://doi.org/10.1245/s10434-018-6462-1. 
24. Love MI, Huber W, Anders S. Moderated estimation of fold change and dispersion for RNA-seq data with DESeq2. Genome Biol. 2014;15(12):550. https://doi.org/10.1186/s13059-014-0550-8.

25. Zhang B, Zhang Z, Li L, Qin YR, Liu H, Jiang C, et al. TSPAN15 interacts with BTRC to promote oesophageal squamous cell carcinoma metastasis via activating NF-kappaB signaling. Nat Commun. 2018;9(1):1423. https://doi. org/10.1038/s41467-018-03716-9

26. Huang J, Yan J, Zhang J, Zhu S, Wang Y, Shi T, et al. SUMO1 modification of PTEN regulates tumorigenesis by controlling its association with the plasma membrane. Nat Commun. 2012;3(1):911. https://doi.org/10.1038/ncomms1 919.

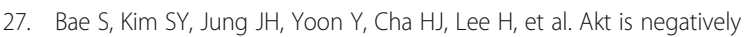
regulated by the MULAN E3 ligase. Cell Res. 2012;22(5):873-85. https://doi. org/10.1038/cr.2012.38.

28. Liao Y, Liu N, Xia X, Guo Z, Li Y, Jiang L, et al. USP10 modulates the SKP2/ Bcr-Abl axis via stabilizing SKP2 in chronic myeloid leukemia. Cell Discov. 2019:5(1):24. https://doi.org/10.1038/s41421-019-0092-z.

29. Petrilli $\mathrm{V}$. The multifaceted roles of inflammasome proteins in cancer. Curr Opin Oncol. 2017;29(1):35-40. https://doi.org/10.1097/CCO. 0000000000000346.

30. Barbe F, Douglas T, Saleh M. Advances in nod-like receptors (NLR) biology. Cytokine Growth Factor Rev. 2014;25(6):681-97. https://doi.org/10.1016/j. cytogfr.2014.07.001.

31. Nagarsheth N, Wicha MS, Zou W. Chemokines in the cancer microenvironment and their relevance in cancer immunotherapy. Nat Rev Immunol. 2017;17(9):559-72. https://doi.org/10.1038/nri.2017.49.

32. Colegio OR, Chu NQ, Szabo AL, Chu T, Rhebergen AM, Jairam V, et al. Functional polarization of tumour-associated macrophages by tumourderived lactic acid. Nature. 2014;513(7519):559-63. https://doi.org/10.1038/ nature13490.

33. Messaed C, Chebaro W, Di Roberto RB, Rittore C, Cheung A, Arseneau J, et al. NLRP7 in the spectrum of reproductive wastage: rare nonsynonymous variants confer genetic susceptibility to recurrent reproductive wastage. J Med Genet. 2011;48(8):540-8. https://doi.org/10.1136/jmg.2011. 089144.

34. Clague MJ, Urbe S. Ubiquitin: same molecule, different degradation pathways. Cell. 2010;143(5):682-5. https://doi.org/10.1016/j.cell.2010.11.012.

35. Bednash JS, Weathington N, Londino J, Rojas M, Gulick DL, Fort R, et al. Targeting the deubiquitinase STAMBP inhibits NALP7 inflammasome activity. Nat Commun. 2017;8(1):15203. https://doi.org/10.1038/ncomms152 03.

36. Martinez-Torres RJ, Chamaillard M. The ubiquitin code of NODs signaling pathways in health and disease. Front Immunol. 2019;10:2648. https://doi. org/10.3389/fimmu.2019.02648.

37. Ver Heul AM, Fowler CA, Ramaswamy S, Piper RC. Ubiquitin regulates caspase recruitment domain-mediated signaling by nucleotide-binding oligomerization domain-containing proteins NOD1 and NOD2. J Biol Chem. 2013;288(10):6890-902. https://doi.org/10.1074/jbc.M112.413781.

38. Han S, Lear TB, Jerome JA, Rajbhandari S, Snavely CA, Gulick DL, et al. Lipopolysaccharide primes the NALP3 Inflammasome by inhibiting its Ubiquitination and degradation mediated by the SCFFBXL2 E3 ligase. J Biol Chem. 2015;290(29):18124-33. https://doi.org/10.1074/jbc.M115.645549.

39. Py BF, Kim MS, Vakifahmetoglu-Norberg H, Yuan J. Deubiquitination of NLRP3 by BRCC3 critically regulates inflammasome activity. Mol Cell. 2013; 49(2):331-8. https://doi.org/10.1016/j.molcel.2012.11.009.

40. Zhu H, Yan F, Yuan T, Qian M, Zhou T, Dai X, et al. USP10 promotes proliferation of hepatocellular carcinoma by Deubiquitinating and stabilizing YAP/TAZ. Cancer Res. 2020;80(11):2204-16. https://doi.org/10.1158/0008-54 72.CAN-19-2388

41. Lu C, Ning Z, Wang A, Chen D, Liu X, Xia T, et al. USP10 suppresses tumor progression by inhibiting mTOR activation in hepatocellular carcinoma. Cancer Lett. 2018;436:139-48. https://doi.org/10.1016/j.canlet.2018.07.032.

42. Vitale I, Manic G, Coussens LM, Kroemer G, Galluzzi L. Macrophages and metabolism in the tumor microenvironment. Cell Metab. 2019;30(1):36-50. https://doi.org/10.1016/j.cmet.2019.06.001

43. Qian BZ, Pollard JW. Macrophage diversity enhances tumor progression and metastasis. Cell. 2010;141(1):39-51. https://doi.org/10.1016/j.cell.2010.03.014.

44. Lin $Y, X u J$, Lan $H$. Tumor-associated macrophages in tumor metastasis: biological roles and clinical therapeutic applications. J Hematol Oncol. 2019; 12(1):76. https://doi.org/10.1186/s13045-019-0760-3.
45. Yang $H$, Zhang Q, Xu M, Wang L, Chen X, Feng Y, et al. CCL2-CCR2 axis recruits tumor associated macrophages to induce immune evasion through PD-1 signaling in esophageal carcinogenesis. Mol Cancer. 2020;19(1):41. https://doi.org/10.1186/s12943-020-01165-X.

46. Li X, Yao W, Yuan Y, Chen P, Li B, Li J, et al. Targeting of tumour-infiltrating macrophages via CCL2/CCR2 signalling as a therapeutic strategy against hepatocellular carcinoma. Gut. 2017;66(1):157-67. https://doi.org/10.1136/ gutjnl-2015-310514.

47. Lupfer C, Kanneganti TD. Unsolved mysteries in NLR biology. Front Immunol. 2013;4:285.

48. Girardin SE, Tournebize R, Mavris M, Page AL, Li X, Stark GR, et al. CARD4/ Nod1 mediates NF-kappaB and JNK activation by invasive Shigella flexneri. EMBO Rep. 2001;2(8):736-42. https://doi.org/10.1093/embo-reports/kve155.

49. Li X, Wang M, Gong T, Lei X, Hu T, Tian M, et al. A S100A14-CCL2/CXCL5 signaling axis drives breast cancer metastasis. Theranostics. 2020;10(13): 5687-703. https://doi.org/10.7150/thno.42087.

\section{Publisher's Note}

Springer Nature remains neutral with regard to jurisdictional claims in published maps and institutional affiliations.
Ready to submit your research? Choose BMC and benefit from:

- fast, convenient online submission

- thorough peer review by experienced researchers in your field

- rapid publication on acceptance

- support for research data, including large and complex data types

- gold Open Access which fosters wider collaboration and increased citations

- maximum visibility for your research: over $100 \mathrm{M}$ website views per year

At $\mathrm{BMC}$, research is always in progress.

Learn more biomedcentral.com/submissions 